\title{
Average-Position Coordination for Distributed Multi-User Networked Haptic Cooperation
}

\author{
Ramtin Rakhsha and Daniela Constantinescu \\ Assistive Robotic and Mechatronics System Lab (ARMLab), \\ Department of Mechanical Engineering, University of Victoria, BC, Canada
}

Proportional-derivative (PD) control is often used to coordinate the two copies of the virtual environment in distributed two-users networked haptic cooperation. However, a distributed PD controller designed for force interactions between two users may destabilize the haptic cooperation among multiple users because the effective coordination gain for each local copy of the virtual environment increases with the participant count. This paper proposes the average position (AP) strategy to upper bound the effective stiffness for the shared virtual object (SVO) coordination and, thus, to increase the stability of distributed multi-user haptic cooperation. The paper first motivates the AP strategy via continuous-time analysis of the autonomous dynamics of an SVO distributed among $N$ users connected across a network with infinite bandwidth and no communication delay. We then investigate the effect of AP coordination on distributed multi-user haptic interactions over a network with limited bandwidth and constant and small communication delay via multi-rate stability and performance analyses of cooperative manipulations of an SVO by up to five operators. The paper shows that AP coordination: (1) has bounded effective coordination gain; (2) increases the stability region of distributed multi-user haptic cooperation compared to conventional PD coordination; and (3) renders less viscous SVO dynamics to operators than PD coordination. Three-users experimental manipulations of a shared virtual cube validate the analysis.

Keywords: networked haptic cooperation, distributed control, multi-rate control, average-position scheme

\section{Introduction}

The rapid growth of computer networks over the past decade has provided the impetus for adding the sense of touch to applications, such as cooperative industrial design (Glencross et al., 2007), surgical teletraining (Mitra \& Niemeyer, 2007), telerehabilitation (Sugarman, Dayan, Weisel-Eichler, \& Tiran, 2006), and multi-user on-line computer games (Kim, Han, Kim, Kim, \& Song, 2007). In these applications, multiple distant users must interact with other users and/or with the shared virtual environment simultaneously.

Haptic sharing of virtual environments can be supported through centralized control and clientserver communications (I. Lee \& Choi, 2009; S. Lee \& Kim, 2009), or through distributed control

\footnotetext{
Authors retain copyright and grant the Journal of Human-Robot Interaction right of first publication with the work simultaneously licensed under a Creative Commons Attribution License that allows others to share the work with an acknowledgement of the work's authorship and initial publication in this journal.
}

Journal of Human-Robot Interaction, Vol. 4, No. 3, 2015, Pages 62-75. DOI 10.5898/JHRI.4.3.Rakhsha 
and peer-to-peer communications (Fotoohi, Sirouspour, \& Capson, 2007; Huang \& Lee, 2013). In the client-server architecture, users send their motion information to a central server that simulates the virtual environment and returns interaction forces to users. In the peer-to-peer paradigm, each user simulates a local copy of the virtual environment and sends their motion information with other peers. Peer-to-peer architectures are often preferred because they introduce only half of the communication delay of client-server architectures in the force feedback loop, and, as a result, they can support haptic cooperation in stiffer virtual environments. Therefore, this paper focuses on multi-user haptic cooperation with peer-to-peer communications.

Prior work on collaborative haptic systems can be described in the following categories: (1) communications approaches, which improve the haptic experience in the shared virtual environment through novel network or data transmission protocols (Eid, Cha, \& El-Saddik, 2011; Marshall, Yap, \& Yu, 2008; Schuwerk, Paggetti, Chaudhari, \& Steinbach, 2014; Zhengzheng, Zhan, Xiang, \& Wanjie, 2013); (2) control approaches, which provide stability and transparency guarantees through new methods to model and control the multiple simultaneous force interactions; and (3) experimental studies, which validate communication and control approaches through implementation on various testbeds (Basdogan, Ho, Srinivasan, \& Slater, 2000; Buttolo, Oboe, \& Hannaford, 1997; Gunn, Hutchins, \& Adcock, 2005; Qin, Choi, Xu, Pang, \& Heng, 2013; Sankaranarayanan \& Hannaford, 2006). Because this paper addresses the coordination control of a distributed shared virtual object (SVO), it discusses prior control methods for multi-user haptic cooperation in more detail.

Many existing control approaches stabilize the multi-user haptic cooperation via passivity-based conditions on PD-type control gains. In a centralized setting, a state-space passivity condition in the form of linear matrix inequalities is employed to ensure the stability of multi-user haptic interactions (Bianchini, Orlandesi, \& Prattichizzo, 2010). Virtual coupling parameters are computed to guarantee the stability of the cooperative haptic system for a connectivity known a priori of a centralized SVO. However, neither experimental validation nor performance analysis are presented. A modified passive-set-position-modulation algorithm (Lee \& Huang, 2010) on a centralized haptic cooperation system was implemented (Ansari, Zareinejad, Rezaei, Baghestan, \& Sarli, 2012). The passive-setposition-modulation approach requires local injection of damping into the SVO dynamics that may result to poor performance and impose sluggish motion of the SVOs. In a decentralized study, a distributed control framework for $N$-users haptic collaboration was proposed (Cheong, Niculescu, $\& \mathrm{Kim}, 2009)$ that demanded ring-type network connectivity and a priori knowledge of the system dynamics and of the connectivity sequence. Huang and Lee (2013) introduced a discrete-time passivity framework to asymptotically synchronize $N$ copies of a distributed SVO with second order discrete-time dynamics. They formulated the SVO synchronization as a discrete-time second-order consensus problem and employed discrete-time passive integrators (Lee \& Huang, 2008) to design a passive peer-to-peer control architecture. Their passivity condition relies on local damping injection at each user location and thus makes the technique conservative for virtual environments where little local SVO damping is of interest. Passivity-based conditions on multi-rate multi-user haptic cooperation with wave domain communications were derived in a centralized setting (Yasrebi \& Constantinescu, 2009) and were used in a distributed setting (Rakhsha \& Constantinescu, 2012). Analysis and experiments in those works showed that the passive multi-rate wave communications could maintain multi-user haptic interaction stability regardless of the magnitude of fixed communication delays. Absolute stability conditions on PD-type control gains were presented (Khademian \& Hashtrudi-Zaad, 2011; Li, Tavakoli, \& Huang, 2014a) for trilateral haptic systems, and recently (Li, Tavakoli, \& Huang, 2014b), for multi-user haptic systems.

Although PD control is often used to coordinate the two copies of the SVO in distributed twouser networked haptic cooperation, its direct application to haptic interaction among multiple users may lead to instability as the effective coordination gain for each local copy of the SVO increases 
with the participant count. This paper proposes the average position (AP) strategy to upper bound the effective stiffness for the shared virtual object (SVO) coordination, and thus, to increase the stability of distributed multi-user haptic cooperation. The paper extends preliminary results (Rakhsha \& Constantinescu, 2011) via analytical and experimental comparisons of the proposed AP and conventional PD schemes. Analysis focuses on multi-user haptic cooperation across a computer network with limited bandwidth and constant communication delays. To support fast sampling of the users' force feedback loops in the presence of slower network update rates, this paper integrates AP coordination into a dual-rate haptic control architecture. In the dual-rate architecture, users' local haptic rendering loops are sampled at the typical force control interval $T_{c}=0.001 \mathrm{~s}$ and receive synchronized updates from the other local feedback loops every $T_{n}=M T_{c}$, where $M$ is a positive integer. This work employs lifting (Araki \& Yamamoto, 1986; Fotoohi et al., 2007; Barbagli, Prattichizzo, \& Salisbury, 2005) to model multi-user networked haptic cooperation with AP coordination and to investigate its stability and performance. This paper analyzes the autonomous dynamics of SVO distribution among $N$ users over a network with infinite bandwidth and no communication delay; investigates the stability and performance of the AP scheme among up to five users over a network with limited bandwidth, small and constant communication delay; and validates the multi-rate stability and performance analyses via experiments in which three users manipulate a virtual cube together.

In the remainder of the paper, Section 2 motivates the proposed AP coordination of a distributed SVO before introducing it and shows that its effective stiffness is upper bounded by the coordination stiffness of two-users cooperation. Section 3 derives the closed-loop state-space dynamics of dualrate, multi-user networked haptic interaction and investigates the stability of force feedback with AP coordination for up to five operators. Section 4 presents the frequency-domain performance of the AP coordination of networked haptic cooperation among up to five users. Section 5 validates that AP coordination increases the stability and the performance of distributed multi-user networked haptic cooperation through experiments in which three networked users manipulate a shared virtual cube together. Section 6 concludes the paper with suggestions for future work.

\section{Average-position coordination}

Distributed multi-user networked haptic cooperation is implemented by providing a local copy of the SVO to each peer user, coordinating all SVO copies through distributed coordination control, and rendering the dynamics and contacts of the local SVO to each user through haptic interaction control. Typically, virtual coupling is selected for haptic rendering and PD controllers are used to coordinate the distributed SVO copies to each other, as depicted in Fig. 1. The mass of the SVO is equally distributed among, and the damping of the SVO is inherited by, all of its copies. Such conventionally distributed PD coordination of the SVO is straightforward to implement, but its effective stiffness grows with the number of users. Therefore, controller gains designed for two-user haptic cooperation destabilize force interactions among multiple operators. This paper proposes the average position (AP) coordination strategy to upper bound the effective SVO coordination gain to the stiffness of two-users coordination.

The gain dependency of conventional PD coordination on the number of cooperating peers can be derived from the autonomous dynamics of SVO distribution across a network with infinite bandwidth and no communication delay. These dynamics are obtained starting from the dynamics of the $i$-th local copy of the distributed SVO:

$$
\frac{m_{\mathrm{O}}}{N} \ddot{x}_{\mathrm{O} i}+b_{\mathrm{O}} \dot{x}_{\mathrm{O} i}=\sum_{j=1, j \neq i}^{N}\left(K_{\mathrm{T}}\left(x_{\mathrm{O} j}-x_{\mathrm{O} i}\right)+B_{\mathrm{T}}\left(\dot{x}_{\mathrm{O} j}-\dot{x}_{\mathrm{O} i}\right)\right),
$$




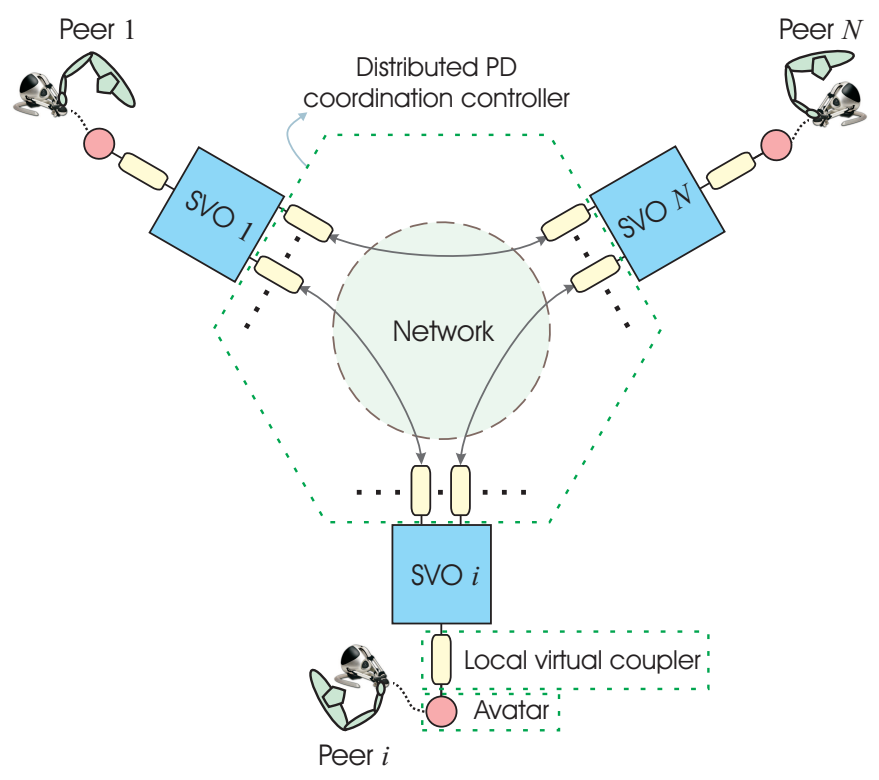

Figure 1. Conventional PD coordination of distributed haptic cooperation among $N$ networked users.

where $m_{\mathrm{O}}$ and $b_{\mathrm{O}}$ are the mass and damping of the SVO, $N$ is the number of users among which the SVO is distributed, $K_{\mathrm{T}}$ and $B_{\mathrm{T}}$ are the stiffness and damping gains of the coordinating PD controller, and $x_{\mathrm{O} l}, \dot{x}_{\mathrm{O} l}, \ddot{x}_{\mathrm{O} l}$ are the position, velocity and acceleration, respectively, of the $l$-th SVO copy, with $l=1, \ldots, N$. Together, the $N$ local SVO dynamics in Eq. (1) yield the autonomous dynamics of the distributed SVO with PD coordination:

$$
\begin{aligned}
\frac{m_{\mathrm{O}}}{N} \mathbf{I}_{N \times N} \ddot{\mathbf{x}}_{\mathrm{O}} & +\left(\left(b_{\mathrm{O}}+N B_{\mathrm{T}}\right) \mathbf{I}_{N \times N}-B_{\mathrm{T}} \mathbf{1}_{N \times 1} \mathbf{1}_{1 \times N}\right) \dot{\mathbf{x}}_{\mathrm{O}} \\
& +\left(N K_{\mathrm{T}} \mathbf{I}_{N \times N}-K_{\mathrm{T}} \mathbf{1}_{N \times 1} \mathbf{1}_{1 \times N}\right) \mathbf{x}_{\mathrm{O}}=\mathbf{0} .
\end{aligned}
$$

In Eq. (2), $\mathbf{x}_{O}, \dot{\mathbf{x}}_{O}$ and $\ddot{\mathbf{x}}_{O}$ are $N$-dimensional vectors that collect the positions, velocities and accelerations of all SVO copies, respectively. $\mathbf{I}_{N \times N}$ is the $N$-dimensional unity matrix and $\mathbf{1}_{r \times c}$ is an $r \times c$ matrix with all entries of 1 . The stiffness matrix of the SVO's PD coordination in Eq. (2) is:

$$
\mathbf{K}_{\mathrm{PD}}=K_{\mathrm{T}}\left(N \mathbf{I}_{N \times N}-\mathbf{1}_{N \times 1} \mathbf{1}_{1 \times N}\right),
$$

and has one zero eigenvalue, which corresponds to the rigid body motion of the SVO, and one eigenvalue with geometric multiplicity $(N-1)$, which is called effective coordination stiffness herein and grows with $N$. To bound the effective coordination stiffness, this paper proposes the AP coordination strategy.

In the AP scheme, each SVO copy is locally coordinated to the average position of all other remote SVO copies, as schematically depicted in Fig. 2 for the SVO copy of Peer $i$. In this figure, $m_{\mathrm{HD} i}$ and $b_{\mathrm{HD} i}$ are the mass and damping of the haptic device, $m_{\mathrm{O} i}=\frac{m_{\mathrm{O}}}{N}$ and $b_{\mathrm{O} i}=b_{\mathrm{O}}$ are the mass and damping of the SVO copy, $K_{\mathrm{C} i}$ and $B_{\mathrm{C} i}$ are the stiffness and damping of the virtual coupler, and $K_{\mathrm{T}}$ and $B_{\mathrm{T}}$ are the stiffness and damping of the distributed AP coordination controller, all at Peer $i$. The autonomous dynamics of the $i$-th local copy of the distributed SVO with AP 


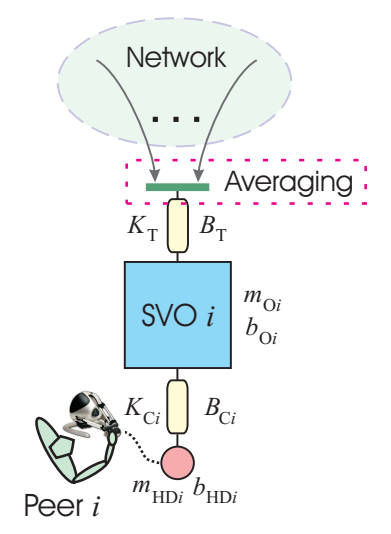

Figure 2. Average-position (AP) coordination of haptic cooperation among $N$ users as applied at Peer $i$.

coordination are:

$$
\begin{aligned}
\frac{m_{\mathrm{O}}}{N} \ddot{x}_{\mathrm{O} i}+b_{\mathrm{O}} \dot{x}_{\mathrm{O} i} & =K_{\mathrm{T}}\left(\frac{\sum_{j=1, j \neq i}^{N} x_{\mathrm{O} j}}{N-1}-x_{\mathrm{O} i}\right) \\
& +B_{\mathrm{T}}\left(\frac{\sum_{j=1, j \neq i}^{N} \dot{x}_{\mathrm{O} j}}{N-1}-\dot{x}_{\mathrm{O} i}\right),
\end{aligned}
$$

and, combined with the dynamics of the other local copies, yield the autonomous dynamics of the distributed SVO with AP coordination:

$$
\begin{aligned}
\frac{m_{\mathrm{O}}}{N} \mathbf{I}_{N \times N} \ddot{\mathbf{x}}_{\mathrm{O}} & =\left(\left(b_{\mathrm{O}}+\frac{N B_{\mathrm{T}}}{N-1}\right) \mathbf{I}_{N \times N}-\frac{B_{\mathrm{T}}}{N-1} \mathbf{1}_{N \times 1} \mathbf{1}_{1 \times N}\right) \dot{\mathbf{x}}_{\mathrm{O}} \\
& +K_{\mathrm{T}}\left(\frac{N}{N-1} \mathbf{I}_{N \times N}-\frac{1}{N-1} \mathbf{1}_{N \times 1} \mathbf{1}_{1 \times N}\right) \mathbf{x}_{\mathrm{O}}
\end{aligned}
$$

The stiffness matrix of AP coordination of the SVO is:

$$
\mathbf{K}_{\mathrm{AP}}=K_{\mathrm{T}}\left(\frac{N}{N-1} \mathbf{I}_{N \times N}-\frac{1}{N-1} \mathbf{1}_{N \times 1} \mathbf{1}_{1 \times N}\right),
$$

and has one zero eigenvalue, which corresponds to the rigid body motion of the distributed SVO, and one eigenvalue with geometric multiplicity $(N-1)$, which decreases strictly monotonically to $K_{\mathrm{T}}$ as $N$ grows. Note that the distributed SVO dynamics are controlled by the eigenvalues of $\left(N / m_{\mathrm{O}}\right) \mathbf{K}_{\mathrm{AP}}$, and therefore, the AP scheme needs to be coupled with inversely proportional scaling of $K_{\mathrm{T}}$ to guarantee stable SVO distribution for arbitrary $N$. Although not employed in the work presented in this paper, such coupling is straightforward to implement.

According to Fig. 2, the dynamics of the distributed $N$-users networked haptic cooperation with AP coordination combine the following:

- the dynamics of the $i$-th haptic device:

$$
m_{\mathrm{HD} i} \ddot{x}_{\mathrm{HD} i}+b_{\mathrm{HD} i} \dot{x}_{\mathrm{HD} i}=F_{\mathrm{h} i}-F_{\mathrm{C} i},
$$


- with the dynamics of the $i$-th SVO copy:

$$
m_{\mathrm{O} i} \ddot{x}_{\mathrm{O} i}+b_{\mathrm{O} i} \dot{x}_{\mathrm{O} i}=F_{\mathrm{C} i}-F_{\mathrm{T} i},
$$

for all $i=1, N$. In Equations (7) and (8): $F_{\mathrm{h} i}$ is the force applied by Peer $i$ to their haptic interface; $F_{\mathrm{C} i}$ is the virtual coupling force at Peer $i$ :

$$
F_{\mathrm{C} i}=K_{\mathrm{C} i}\left(x_{\mathrm{HD} i}-x_{\mathrm{O} i}\right)+B_{\mathrm{C} i}\left(\dot{x}_{\mathrm{HD} i}-\dot{x}_{\mathrm{O} i}\right) ;
$$

and $F_{\mathrm{T} i}$ is the force applied by the AP coordination controller on the $i$-th SVO copy:

$$
F_{\mathrm{T} i}=K_{\mathrm{T}}\left(x_{\mathrm{O} i}-x_{\mathrm{O} i_{d}}\right)+B_{\mathrm{T}}\left(\dot{x}_{\mathrm{O} i}-\dot{x}_{\mathrm{O} i_{d}}\right),
$$

with $x_{\mathrm{O} i d_{d}}$ and $\dot{x}_{\mathrm{O} i_{d}}$ being the desired position and velocity of the $i$-th SVO copy, respectively. In the AP strategy, they are computed via the following equations:

$$
x_{\mathrm{O} i_{d}}(t)=\frac{\sum_{j=1, j \neq i}^{N} x_{\mathrm{O} j}\left(t-T_{d}\right)}{N-1}
$$

and

$$
\dot{x}_{\mathrm{O} i_{d}}(t)=\frac{\sum_{j=1, j \neq i}^{N} \dot{x}_{\mathrm{O} j}\left(t-T_{d}\right)}{N-1},
$$

where $T_{d}$ is the communication delay of the network. In this paper, the network delay $T_{d}$ is assumed constant, equal in all communication channels, and an integer multiple $M$ of the network packet update interval $T_{n}, T_{d}=M T_{n}$.

\section{Stability of dual-rate haptic cooperation among up to five users}

Since the typical network update interval is longer than the sampling interval required for realistic haptic rendering, the distributed haptic cooperation among $N$ networked users is a dual rate closedloop system. Its stability is controlled by the eigenvalues of its multi-rate state transition matrix. This matrix is computed using the lifting approach introduced in (Araki \& Yamamoto, 1986) and first applied to haptic cooperation in (Fotoohi et al., 2007). The derivations are succinctly overviewed in this section.

The open-loop continuous-time state-space dynamics of $N$-users networked haptic cooperation combine the dynamics of the users, the haptic interfaces, and the SVO copies, and then group system inputs and outputs into fast and slow sub-vectors (hereafter indicated with the $c$ and $n$ indices, respectively). The inputs comprise the virtual coupling forces, updated at the fast haptic rate (Eq. (9)), and the SVO coordination forces, with components updated both fast and slow (Eq. (10)), grouped into:

$$
\mathbf{u}^{\top}=\left(\begin{array}{ll}
\mathbf{u}_{c}^{\top} & \mathbf{u}_{n}^{\top}
\end{array}\right)^{\top}
$$

where:

$$
\begin{aligned}
& \mathbf{u}_{c}^{\top}=\left(\begin{array}{lllll}
F_{\mathrm{C} 1} & F_{\mathrm{T}_{1}} & \ldots & F_{\mathrm{C} N} & F_{\mathrm{T} N_{c}}
\end{array}\right)^{\top}, \\
& \mathbf{u}_{n}^{\top}=\left(\begin{array}{lll}
F_{\mathrm{T} 1_{n}} & \cdots & F_{\mathrm{T} N_{n}}
\end{array}\right)^{\top}, \\
& F_{\mathrm{T}_{c}}=K_{\mathrm{T}} x_{\mathrm{O} i}+B_{\mathrm{T}} \dot{x}_{\mathrm{O} i},
\end{aligned}
$$

and,

$$
F_{\mathrm{T} i_{n}}=-K_{\mathrm{T}} x_{\mathrm{O} i_{d}}-B_{\mathrm{T}} \dot{x}_{\mathrm{O} i_{d}} .
$$


The state vector comprises the states of all haptic interfaces and SVO copies:

$$
\mathbf{x}^{\top}=\left(\begin{array}{lllll}
\mathbf{x}_{\text {peer }_{1}} & \ldots & \mathbf{x}_{\text {peer }_{i}} & \ldots & \mathbf{x}_{\text {peer }_{N}}
\end{array}\right)^{\top},
$$

where:

$$
\mathbf{x}_{\text {peeri }}^{\top}=\left(\begin{array}{llll}
x_{\mathrm{HD} i} & \dot{x}_{\mathrm{HD} i} & x_{\mathrm{O} i} & \dot{x}_{\mathrm{O} i}
\end{array}\right)^{\top} ; \quad i=1, \ldots, N .
$$

The output vector is:

$$
\mathbf{y}^{\top}=\left(\begin{array}{ll}
\mathbf{y}_{\mathbf{c}}{ }^{\top} & \mathbf{y}_{\mathbf{n}}^{\top}
\end{array}\right)^{\top}
$$

where:

$$
\mathbf{y}_{\mathbf{c}}^{\top}=\mathbf{x}^{\top}
$$

and,

$$
\mathbf{y}_{n}^{\top}=\left(\begin{array}{lllll}
\mathbf{y}_{\text {peer }_{1_{n}}} & \ldots & \mathbf{y}_{\text {peer }_{i_{n}}} & \ldots & \mathbf{y}_{\text {peer }_{N_{n}}}
\end{array}\right)^{\top}
$$

with,

$$
\mathbf{y}_{\text {peer }_{i_{n}}}^{\top}=\left(\begin{array}{ll}
x_{\mathrm{O} i_{d}} & \dot{x}_{\mathrm{O} i_{d}}
\end{array}\right)^{\top} ; \quad i=1, \ldots, N
$$

Hence, the open-loop continuous-time state-space dynamics of $N$-user networked haptic cooperation with AP coordination are as follows:

$$
\begin{aligned}
\dot{\mathbf{x}}_{4 N \times 1} & =\mathbf{A}_{4 N \times 4 N} \mathbf{x}_{4 N \times 1}+\mathbf{B}_{4 N \times 3 N} \mathbf{u}_{3 N \times 1}, \\
\mathbf{y}_{6 N \times 1} & =\mathbf{C}_{6 N \times 4 N} \mathbf{x}_{4 N \times 1}
\end{aligned}
$$

and their discretization is obtained by lifting (Araki \& Yamamoto, 1986), in the form of:

$$
\begin{aligned}
\mathbf{x}_{\mathrm{D}_{(M .4 N) \times 1}}[k+1] & =\mathbf{A}_{\mathrm{D}_{(M .4 N) \times(M .4 N)}} \mathbf{x}_{\mathrm{D}_{(M .4 N) \times 1}}[k] \\
& +\mathbf{B}_{\mathrm{D}_{(M .4 N) \times((2 M+1) . N)}} \mathbf{u}_{\mathbf{D}_{((2 M+1) . N) \times 1}}[k] \\
\mathbf{y}_{\mathrm{D}_{(2 M+1) .2 N \times 1}}[k] & =\hat{\mathbf{C}}_{\mathbf{D}_{((2 M+1) .2 N) \times(M .4 N)}} \mathbf{x}_{\mathrm{D}_{(M .4 N) \times 1}}[k] \\
& +\hat{\mathbf{D}}_{\mathrm{D}_{((2 M+1) .2 N) \times((2 M+1) . N)}} \mathbf{u}_{\mathbf{D}_{((2 M+1) . N) \times 1}}[k]
\end{aligned}
$$

The derivation of $\mathbf{A}_{\mathrm{D}}, \mathbf{B}_{\mathrm{D}}, \hat{\mathbf{C}}_{\mathrm{D}}$ and $\hat{\mathbf{D}}_{\mathrm{D}}$ and the incorporation of communication delays, via augmenting the state with the delayed inputs, are detailed in (Fotoohi et al., 2007).

The stability of the dual-rate distributed $N$-users networked haptic cooperation hinges on the eigenvalues of the closed-loop state transition matrix:

$$
\mathbf{A}_{\mathrm{D}}^{c l}=\mathbf{A}_{\mathrm{D}_{\text {aug }}}+\mathbf{B}_{\mathrm{D}_{\text {aug }}} \mathbf{F}_{\mathrm{D}}\left(\mathbf{I}-\mathbf{D}_{\mathrm{D}_{\text {aug }}} \mathbf{F}_{\mathrm{D}}\right)^{-1} \mathbf{C}_{\mathrm{D}_{\text {aug }}},
$$

where $\mathbf{A}_{\mathrm{D}_{\text {aug }}}, \mathbf{B}_{\mathrm{D}_{\text {aug }}}, \mathbf{C}_{\mathrm{D}_{\text {aug }}}$ and $\mathbf{D}_{\mathrm{D}_{\text {aug }}}$ are the state transition matrices obtained after augmentation with computational and communication delays, and the feedback matrix $\mathbf{F}_{\mathrm{D}}$ includes the virtual coupling and SVO coordination forces. The $\mathbf{F}_{\mathrm{D}}$ is computed by using the approach introduced in (Araki \& Yamamoto, 1986). Namely, the $N$-users haptic cooperation is stable if and only if all eigenvalues of $\mathbf{A}_{\mathrm{D}}^{c l}$ are inside the unit circle.

\section{Stability regions}

This section presents a numerical investigation of the stability regions of dual-rate haptic cooperation among up to five users, with $\mathrm{AP}$ and $\mathrm{PD}$ coordination. The following parameters are used in the following computations: $m_{\mathrm{HD}_{i}}=0.1 \mathrm{~kg}, b_{\mathrm{HD}_{i}}=5.0 \mathrm{Ns} / \mathrm{m} ; m_{O}=0.45 \mathrm{~kg}, b_{O}=0.0 \mathrm{Ns} / \mathrm{m}$, $T_{\mathrm{c}}=0.001 \mathrm{~s}, T_{\mathrm{n}}=0.008 \mathrm{~s}$. The haptic device parameters are selected to approximate the mass 


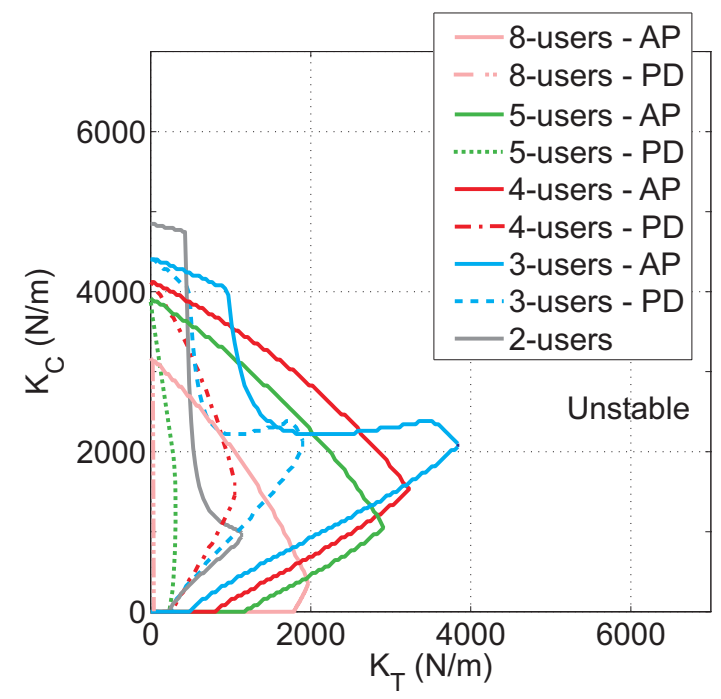

Figure 3. Stability region for haptic cooperation among up to five users with PD coordination, and for haptic cooperation among up to eight users with AP coordination $\left(B_{\mathrm{T}}=0 \mathrm{Ns} / \mathrm{m}\right)$.

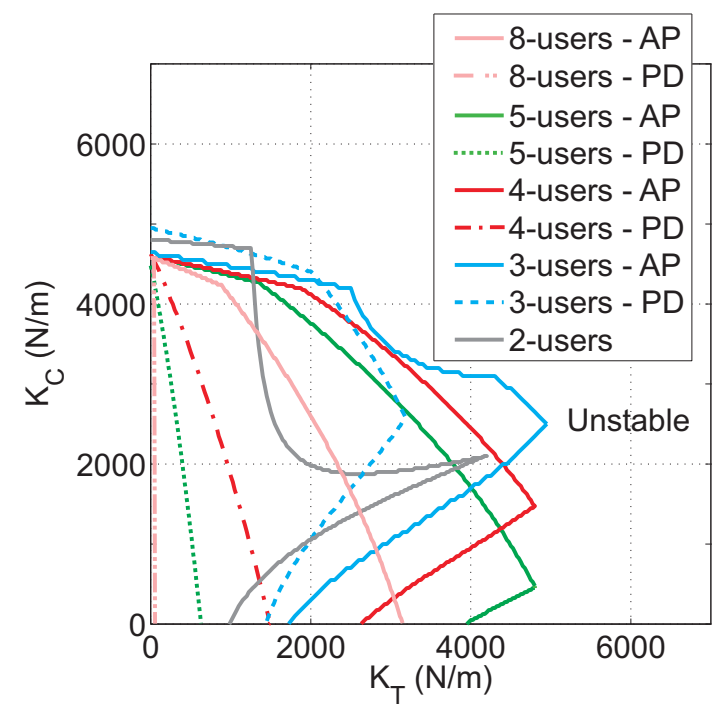

Figure 4. Stability region for haptic cooperation among up to five users with PD coordination, and for haptic cooperation among up to eight users with AP coordination $\left(B_{\mathrm{T}}=5 \mathrm{Ns} / \mathrm{m}\right)$. 
and viscous damping of the Novint devices and the SVO parameters are chosen heuristically. Fig. 3 depicts the stability regions for undamped control, i.e., $B_{\mathrm{T}}=B_{\mathrm{c} i}=0 \mathrm{Ns} / \mathrm{m}$. Fig. 4 shows the stability regions for damped control with $B_{\mathrm{T}}=B_{\mathrm{c} i}=5 \mathrm{Ns} / \mathrm{m}$.

Figs. 3 and 4 show that the proposed AP coordination maintains the stability region of haptic cooperation largely unaffected by the number of interacting users, regardless of damping in the coordination and haptic rendering controllers. This is in contrast to the PD coordination, where stability region decreases as the number of peers grows.

\section{Performance evaluation}

In distributed multi-user haptic cooperation, the position discrepancy among the SVO copies threatens the fidelity of the force interactions. For example, suppose that Peer $i$ tries to move their local copy of the SVO while other users are not in touch with their local copies. If the coordination of the $N$ distributed SVO copies is perfect, Peer $i$ imposes the same motion on all SVO copies and feels the multiple SVO copies as rigidly attached to each other. However, for coordination with limited gains, Peer $i$ feels the SVO copies as connected to each other through springs with finite stiffness and with slowly moving ends (due to the delayed updates received across the network). This section uses the admittance $G_{i}(z)=\frac{F_{h_{i}}(z)}{\dot{x}_{i}(z)}$ of the SVO copies to compare the dynamics of the distributed SVO rendered to users by AP and PD coordination to the dynamics of an "ideal" virtual object. The $G_{i}$-parameters are computed after lifting the dual-rate system to its unirate counterpart.

The frequency responses of a SVO distributed among up to five users and coordinated via the AP and PD schemes are depicted in Fig. 5. The parameter values selected for the analysis are as follows: $m_{\mathrm{O}}=0.45 \mathrm{~kg} ; b_{\mathrm{O}}=0 \mathrm{Ns} / \mathrm{m} ; K_{T}=1000 \mathrm{~N} / \mathrm{m} ; B_{T}=5 \mathrm{Ns} / \mathrm{m}$ respectively; and $T_{d}=T_{n}=0.008 \mathrm{~s}$ in all communication links.

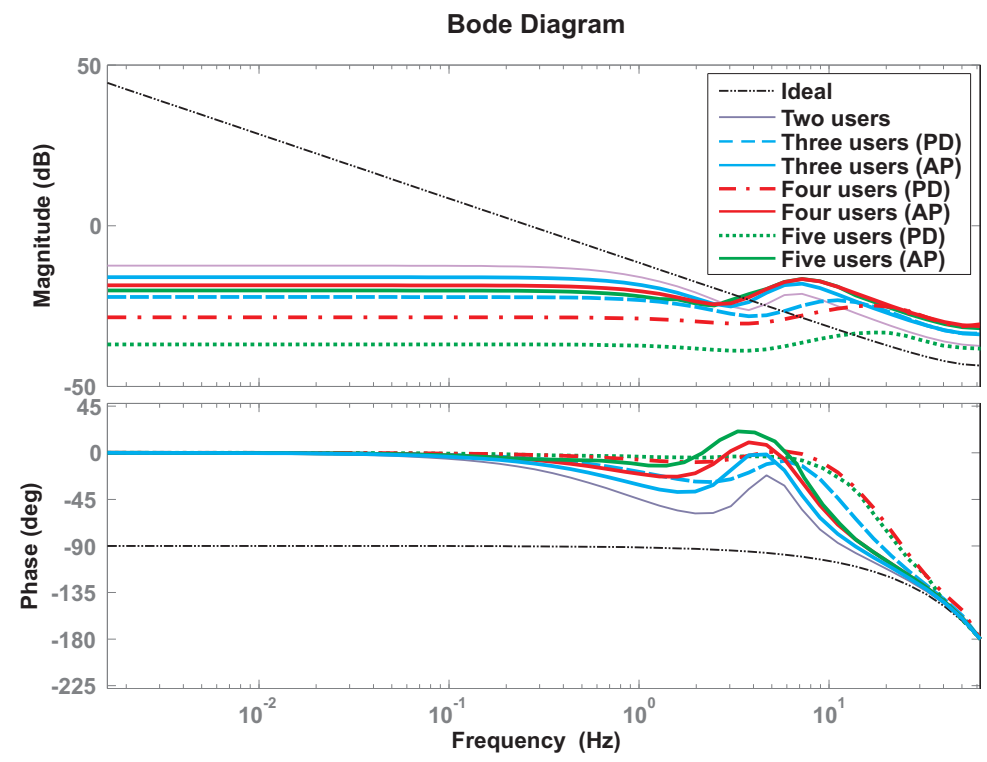

Figure 5. SVO admittance for haptic cooperation among up to five users, with AP and PD coordination, and with communication delay $T_{d}=T_{n}=0.008 \mathrm{~s}$ in all links.

The deviations from the ideal frequency response show that the distributed SVO has larger viscous damping than the "ideal" SVO, due to the low packet update rate, delays in the communication 
channel, and the damping of the coordination (Fotoohi et al., 2007). Nonetheless, Fig. 5 confirms that: (1) a distributed SVO with AP coordination has admittance closer to the admittance of a pure mass than a distributed SVO with PD coordination, and (2) damping of the distributed SVO rendered to users increases with peer count. This is expected given that the number of communication links increases, as each link introduces additional damping in the cooperation.

\section{Experimental validation}

This section validates the analytical results in the sections above through experiments performed on a testbed that comprises three Falcon Novint haptic devices connected to three computers. The computers all run Windows XP on an Intel Core 2 Duo CPU at $2.67 \mathrm{Ghz}$ with 2 GB RAM, and communicate over a local area network via the UDP protocol with a data transmission rate of $125 \mathrm{~Hz}$. The virtual environment is simulated at $1 \mathrm{kHz}$ via a $\mathrm{C}^{++}$console application and includes a shared virtual cube constrained by a virtual enclosure to move along a single horizontal $x$-direction, and three virtual spheres representing the haptic devices. Given the proximity of the three computers, the actual network delay is negligible. Therefore, a Wide Area Network Emulator (WANem) is used to implement a desired network delay $T_{d}$, equal in all communication channels. Fig. 6 depicts the experimental testbed with three cooperating users.

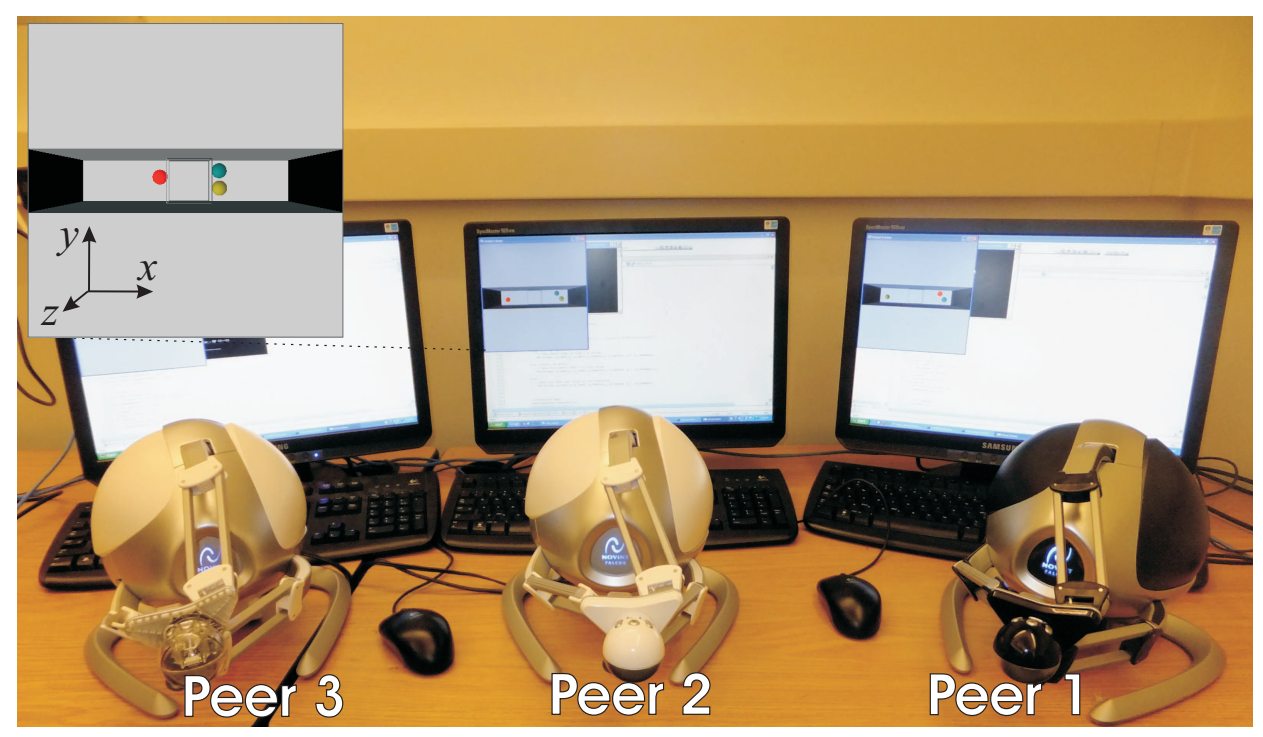

Figure 6. Experimental testbed.

In all experiments, the parameter values are as follows: The mass and damping of the SVO are $m_{\mathrm{O}}=0.45 \mathrm{~kg}$; and $b_{\mathrm{O}}=0 \mathrm{Ns} / \mathrm{m}$, respectively; the coordination damping is $B_{T}=5 \mathrm{Ns} / \mathrm{m}$; the virtual coupling stiffness and damping are $K_{C}=3500 \mathrm{~N} / \mathrm{m}$ and $B_{C}=5 \mathrm{Ns} / \mathrm{m}$, respectively; and the round-trip network delay is $T_{d}=0.048 \mathrm{~s}$.

\subsection{Stability tests}

In the experiments carried out in this section, two of the three users cooperatively manipulate the SVO by moving it back and forth along the rectilinear enclosure. The third user is present in the shared virtual environment, but is not in contact with the SVO. The two users who manipulate the SVO are instructed to keep it moving, without concern for the resultant motion. Nonetheless, 
because of the small workspace of the Novint haptic devices, the two users take frequent turns at pushing the SVO, and the resulting SVO motion appears sinusoidal.

The coordination stiffness $K_{\mathrm{T}}$ is selected to verify that AP coordination increases the stability region of distributed three-users haptic cooperation as compared to PD coordination. The empirically chosen numerical value is $K_{\mathrm{T}}=2100 \mathrm{~N} / \mathrm{m}$. For $K_{\mathrm{T}}$ as specified, the experimental results show that haptic cooperation is unstable with PD coordination (Fig. 7a) and is stable with AP coordination (Fig. 7b). Hence, the results in Fig. 7 confirm that, compared to the PD scheme, the AP strategy can keep the distributed three-user haptic cooperation stable for stiffer coordination.
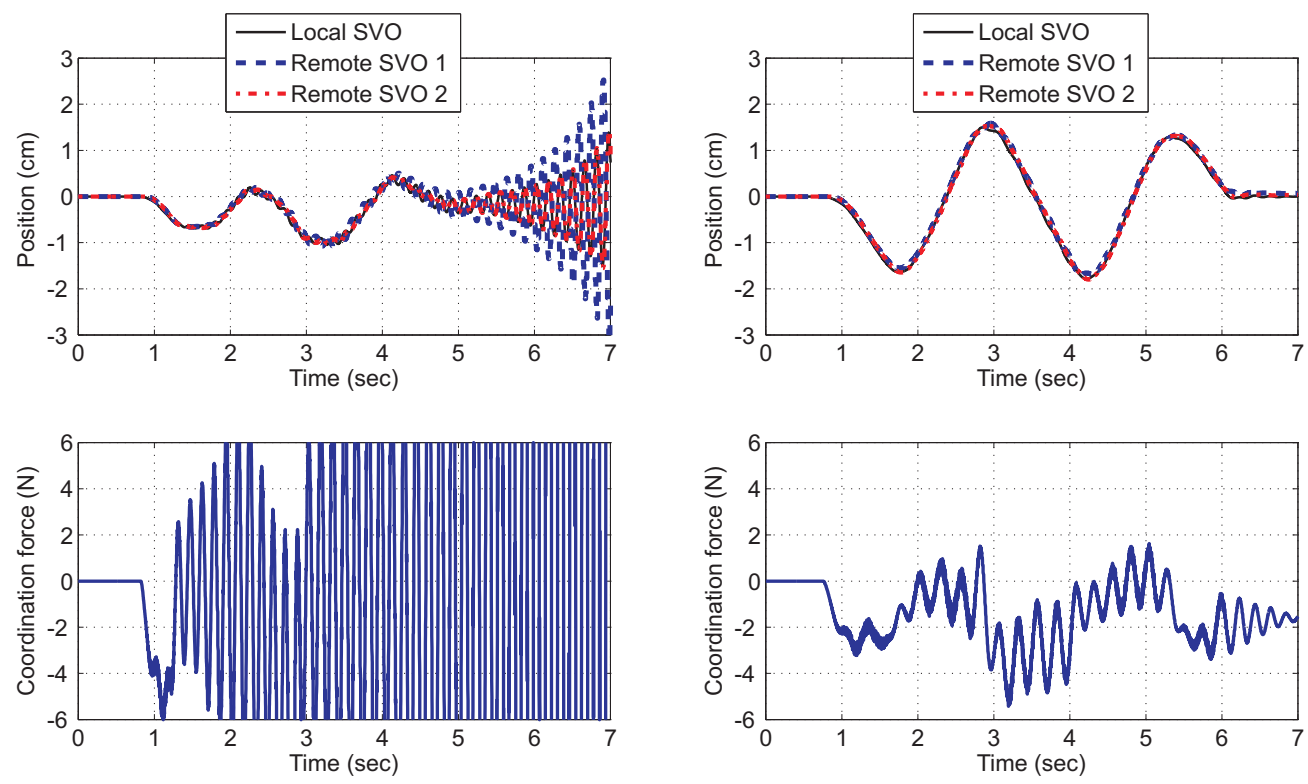

(a) PD coordination.

(b) AP coordination

Figure 7. Experimental three-users haptic cooperation $\left(K_{\mathrm{T}}=2100 \mathrm{~N} / \mathrm{m}\right)$. Note that the coordination forces appear to grow unbounded faster than the SVO positions for PD coordination only because of the plot ranges. In turn, these ranges are chosen to permit the comparative evaluation of the PD and AP controllers' effects on the haptic interaction.

\subsection{Perceived viscosity tests}

This section contrasts the performance of AP coordination to the performance of conventional PD coordination through controlled experiments. The coordination stiffness is set to $K_{T}=1000 \mathrm{~N} / \mathrm{m}$, to ensure that the three-users haptic cooperation is stable both for the PD and for the AP coordination schemes. To guarantee the same initial conditions during successive experiments, users are replaced by controlled forces applied to the haptic devices via commands sent to the servo motors.

At the beginning of each experiment, Peer 1 is at the right end of the rectilinear virtual enclosure and pushes the SVO with a constant force $F_{\mathrm{h} 1}=1.5 \mathrm{~N}$. Peers 2 and 3 are not in contact with the SVO. Thus, the SVO travels along the enclosure to the left during the experiment. Fig. 8 depicting the SVO position at the Peer 1 site shows the following: the SVO reaches a constant velocity when 
pushed by the constant $F_{\mathrm{h} 1}=1.5 \mathrm{~N}$, regardless whether AP or PD coordination is used (i.e., both the AP and PD controllers render a viscously damped SVO to users), and the SVO moves $20 \mathrm{~mm}$ in $1.22 \mathrm{~s}$ (its velocity saturates slower) under AP coordination, and in $1.514 \mathrm{~s}$ (its velocity saturates faster) under PD coordination, (i.e., the AP controller renders a less viscous SVO than the PD controller), as predicted analytically.
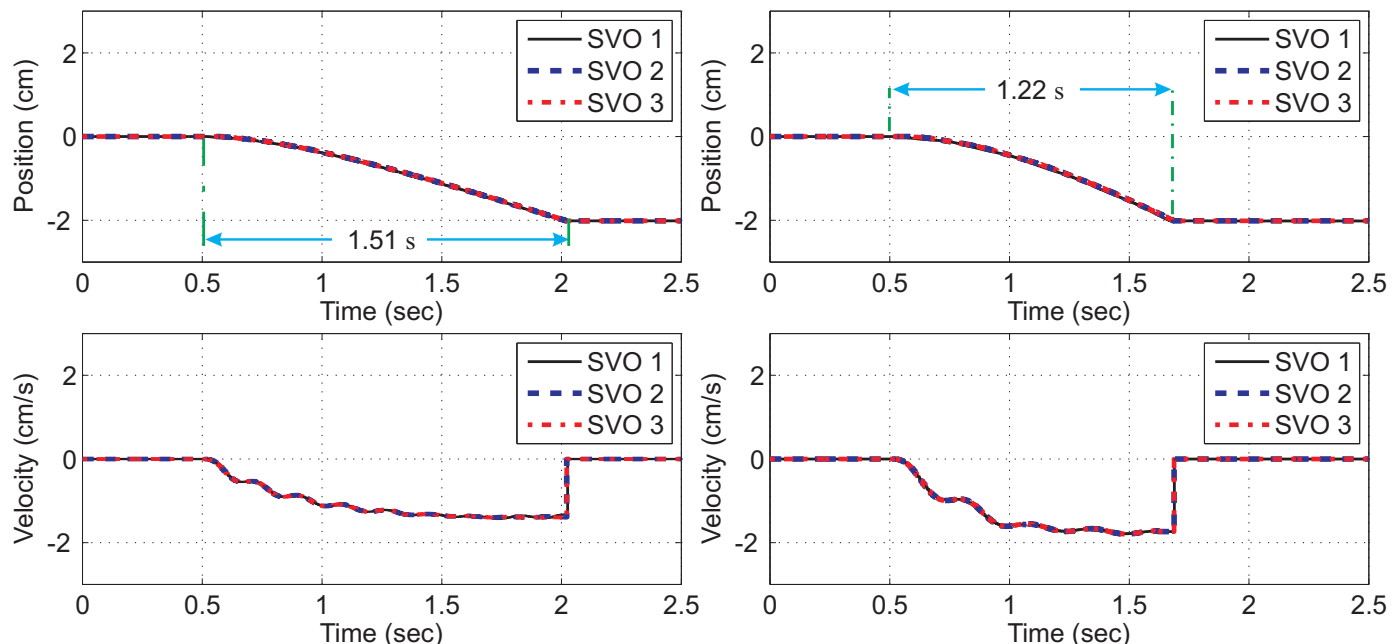

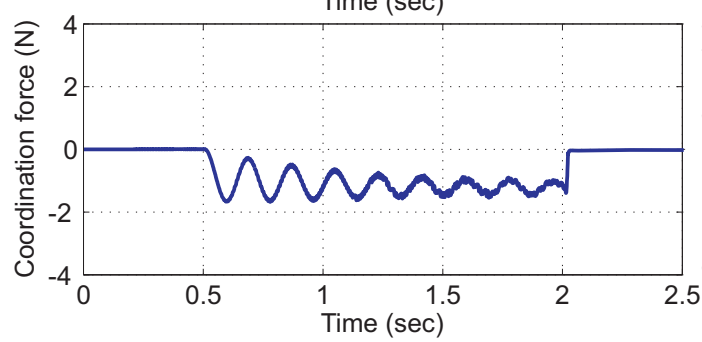

(a) PD coordination.

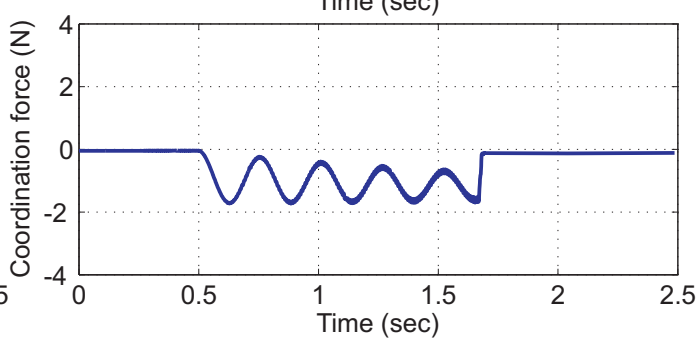

(b) AP coordination

Figure 8. Experimental three-users haptic cooperation with communication delay $T_{d}=6 T_{n}=0.048 \mathrm{~s}$.

\section{Conclusions}

This paper has proposed the average position (AP) coordination for distributed multi-user networked haptic cooperation. Through continuous-time analysis of the autonomous dynamics of an SVO distributed among $N$ users, as well as through multirate analysis of the closed-loop state-space dynamics of up to five-users haptic cooperation, this paper has shown that the AP strategy bounds the coordination gain of the SVO, and thus, increases the stability region of distributed multi-user haptic cooperation as compared to conventional PD coordination. Using numerical analysis, this paper has illustrated that AP coordination is also beneficial to cooperation transparency. Namely, the AP strategy injects less damping in the local feedback loops and therefore renders the SVO dynamics more accurately than (and enhances the performance of the interaction compared to) conventional PD coordination. Experiments in which three users manipulate a shared virtual cube together have validated the analytical results. 
Upcoming work will study the performance of AP coordination in virtual environments relevant to applications, such as multi-user haptic computer games for tele-rehabilitation and education. In these applications, haptic tasks will be defined in collaboration with therapists and/or educators to help cooperating users achieve specific therapeutic and/or educational goals. Future work will also investigate techniques for guaranteeing the stability of distributed haptic cooperation among an arbitrary number of users, across networks with varying communication delay and packet loss, and for SVO with coupled multi degrees-of-freedom dynamics.

\section{Acknowledgements}

This work was supported through an NSERC Discovery Grant.

\section{References}

Ansari, R., Zareinejad, M., Rezaei, S., Baghestan, K., \& Sarli, N. (2012). Stable multi-user interaction with cooperative haptic virtual environments by a modification of passive set-position modulation. IET Control Theory Applications, 6(16), 2538-2548. doi:10.1049/iet-cta.2011.0446

Araki, M., \& Yamamoto, K. (1986). Multivariable multirate sampled-data systems: state-space description, transfer characteristics, and nyquist criterion. IEEE Transactions on Automatic Control, 31(2), 145-154. doi:10.1109/TAC.1986.1104205

Barbagli, F., Prattichizzo, D., \& Salisbury, K. (2005). A multirate approach to haptic interaction with deformable objects with single and multipoint contacts. International Journal of Robotics Research, 24(9), 703-715. doi:10.1177/0278364905057055

Basdogan, C., Ho, C.-H., Srinivasan, M. A., \& Slater, M. (2000). An experimental study on the role of touch in shared virtual environments. ACM Transactions on Computer-Human Interaction, 7(4), 443-460. doi: $10.1145 / 365058.365082$

Bianchini, G., Orlandesi, M., \& Prattichizzo, D. (2010). Passivity-based analysis and design of multi-contact haptic systems via LMIs. In M. Hosseini Zadeh (Ed.), Advances in haptics (p. 155-170). InTech. doi: $10.5772 / 8681$

Buttolo, P., Oboe, R., \& Hannaford, B. (1997). Architectures for shared haptic virtual environments. Computers and Graphics, 21(4), 421-429. doi:10.1016/S0097-8493(97)00019-8

Cheong, J., Niculescu, S.-I., \& Kim, C. (2009). Motion synchronization control of distributed multisubsystems with invariant local natural dynamics. IEEE Transactions on Robotics, 25(2), 382-398. doi: 10.1109/TRO.2008.2011529

Eid, M., Cha, J., \& El-Saddik, A. (2011). Admux: An adaptive multiplexer for haptic-audio-visual data communication. IEEE Transactions on Instrumentation and Measurement, 60(1), 21-31. doi: 10.1109/TIM.2010.2065530

Fotoohi, M., Sirouspour, S., \& Capson, D. (2007). Stability and performance analysis of centralized and distributed multi-rate control architectures for multi-user haptic interaction. International Journal of Robotics Research, 26(9), 977-994. doi:10.1177/0278364907082049

Glencross, M., Jay, C., Feasel, J., Kohli, L., Whitton, M., \& Hubbold, R. (2007). Effective cooperative haptic interaction over the internet. In Proceedings of the IEEE Virtual Reality Conference (p. 115-122). Charlotte, NC. doi:10.1109/VR.2007.352471

Gunn, C., Hutchins, M., \& Adcock, M. (2005). Combating latency in haptic collaborative virtual environments. Presence: Teleoperators and Virtual Environments, 14(3), 313-328. doi:10.1162/105474605323384663

Huang, K., \& Lee, D. J. (2013). Consensus-based peer-to-peer control architecture for multiuser haptic interaction over the internet. IEEE Transactions on Robotics, 29(2), 417-431. doi:10.1109/TRO.2012.2229672

Khademian, B., \& Hashtrudi-Zaad, K. (2011). Shared control architectures for haptic training: Performance and coupled stability analysis. International Journal of Robotics Research, 30(13), 1627-1642. doi: $10.1177 / 0278364910397559$

Kim, Y. B., Han, S. H., Kim, S. J., Kim, H. J., \& Song, C. G. (2007). Multi-player virtual ping-pong game. In Proceedings of the 17th ICAT International Conference on Artificial Reality and Telexistence (p. 269 -273). doi:10.1109/ICAT.2007.34 
Lee, \& Huang. (2008). On passive non-iterative variable-step numerical integration of mechanical systems for haptic rendering. In Proceedings of the ASME Conference of Dynamic Systems and Control Division (p. 1147-1154). doi:10.1115/DSCC2008-2257

Lee, \& Huang. (2010). Passive-set-position-modulation framework for interactive robotic systems. IEEE Transactions on Robotics, 26(2), 354-369. doi:10.1109/TRO.2010.2041877

Lee, I., \& Choi, S. (2009). Discrimination of visual and haptic rendering delays in networked environments. International Journal of Control, Automation and Systems, 7(1), 25-31. doi:10.1007/s12555-009-01040

Lee, S., \& Kim, J. W. (2009). Transparency analysis and delay compensation scheme for hapticbased networked virtual environments. Computer Communications, 32(5), 992-999. doi: 10.1016/j.comcom.2008.12.028

Li, J., Tavakoli, M., \& Huang, Q. (2014a). Absolute stability of a class of trilateral haptic systems. IEEE Transactions on Haptics, 7(3), 301-310. doi:10.1109/TOH.2014.2321616

Li, J., Tavakoli, M., \& Huang, Q. (2014b). Absolute stability of multi-dof multi-lateral haptic systems. IEEE Transactions on Control Systems Technology. doi:10.1109/TCST.2014.2301840

Marshall, A., Yap, K. M., \& Yu, W. (2008). Providing qos for networked peers in distributed haptic virtual environments. Advances in Multimedia, 2008. doi:10.1155/2008/841590

Mitra, P., \& Niemeyer, G. (2007). Haptic simulation of manipulator collisions using dynamic proxies. Presence: Teleoperators and Virtual Environments, 16(4), 367-384. doi:10.1162/pres.16.4.367

Qin, J., Choi, K.-S., Xu, R., Pang, W.-M., \& Heng, P.-A. (2013). Effect of packet loss on collaborative haptic interactions in networked virtual environments: An experimental study. Presence: Teleoperators and Virtual Environments, 22(1), 36-53. doi:10.1162/PRES.a.00132

Rakhsha, R., \& Constantinescu, D. (2011). Enhanced stability of three-users multirate distributed haptic cooperation via coordination to average peer position. In Proceedings of the 4th International Conference on Advances in Computer-Human Interactions (p. 136-141). Gosier, Guadeloupe, France.

Rakhsha, R., \& Constantinescu, D. (2012). On distributed multirate control of direct user-to-user touch in networked haptic systems with passive wave-domain communications. International Journal of Advanced Robotic Systems, 9(52), 1-10. doi:10.5772/50378

Sankaranarayanan, G., \& Hannaford, B. (2006). Virtual coupling schemes for position coherency in networked haptic environments. In Proceedings of the 1st IEEE/RAS-EMBS International Conference on Biomedical Robotics and Biomechatronics (p. 853-858). Pisa, Italy. doi:10.1109/BIOROB.2006.1639197

Schuwerk, C., Paggetti, G., Chaudhari, R., \& Steinbach, E. (2014). Perception-based traffic control for shared haptic virtual environments. Presence: Teleoperators and Virtual Environments, 23(3), 320-338. doi: 10.1162/PRES.a.00196

Sugarman, H., Dayan, E., Weisel-Eichler, A., \& Tiran, J. (2006). The Jerusalem Telerehabilitation System, a new, low-cost, haptic rehabilitation approach. CyberPsychology \& Behavior, 9(2), 178-182. doi: 10.1089/cpb.2006.9.178

Yasrebi, N., \& Constantinescu, D. (2009). Centralized multi-user multi-rate haptic cooperation using wave transformation. In Proceedings of the IEEE International Conference on Mechatronics and Automation (p. 3816-3821). doi:10.1109/ICMA.2009.5246758

Zhengzheng, J., Zhan, G., Xiang, C., \& Wanjie, S. (2013). Remote haptic collaboration for virtual training of lumbar puncture. Journal of Computers, 8(12), 3103-3110. doi:10.4304/jcp.8.12.3103-3110

Authors' names and contact information: Ramtin Rakhsha, Department of Mechanical Engineering, University of Victoria, BC, Canada. Email: rrakhsha@uvic.ca. Daniela Constantinescu, Department of Mechanical Engineering, University of Victoria, BC, Canada. Email: danielac@uvic.ca. 